\title{
Extracting Buildings by Using the Generalized Multi Directional Discrete Radon Transform
}

\author{
I. ELouedi ${ }^{1}$, A. Hamouda ${ }^{1}$, H. Rojbani ${ }^{1}$, R. Fournier ${ }^{2}$, and A. Nait-Ali ${ }^{2}$ \\ ${ }^{1}$ The Sciences institute, Computer technology department, Tunis, Tunisia \\ ineselouedi@yahoo.com, atef_hammouda@yahoo.fr, \\ hmida.rjbagmail.com \\ ${ }^{2}$ The University Paris Est-Creteil, Laboratory of Images, \\ Signal and Intelligent systems(LISSI), Paris, France \\ \{rfournier, naitali\}@u-pec.fr
}

\begin{abstract}
This paper presents a new method to detect and accurately locate a rectangular form object in any given image. In order to find the right coordinates of those objects in the image, we develop the Generalized Multi Directional Discrete Radon Transform (GMDRT). The GMDRT can detect any given shape whatever its form and orientation are. Experimental results on high resolution QuickBird image to extract rectangular buildings form show the efficiency of our method.
\end{abstract}

Keywords: Generalized multi Directional Discrete Radon Transform, HighResolution QuickBird images, Rectangular Buildings.

\section{Introduction}

The recognition of objects whatever its sizes, scales, positions or orientations in images like humans do, is still a challenge for computer vision systems. In recent years, the Radon Transform has received much attention. This transform projects a two dimensional image along straight lines within different directions and then transforms the image into a parameters space where each line in the initial image gives a peak positioned at the corresponding line parameters [1]. This have lead to many line detection applications on image processing [3, 4,8], medical imaging [5] and seismic applications [2]. The Radon Transform was also widely used in satellite image area such as ship wakes detection [5,6], or buildings detection[14].

Especially, the authors in [9] extract buildings from high resolution images by applying the classical Radon Transform. Then, they use the Forstner operator in the Radon transform parameters space to detect peaks. The inconvenience of this approach is its dependence on the buildings size to detect peaks. In addition, this method needs a post-treatment to extract building contours due to the use of the classical Radon Transform which detects only lines.

Here in, we use our Generalized Multi Directional Discrete Radon Transform (GMDRT) to extract directly the rectangular form buildings from an 
image. The extraction problem is reduced only to peaks selection without any posttreatment. The paper is outlined as follows: Section 2 presents the definition of the GMDRT approach. Then, section3 details the process of the buildings extraction from high resolution images. Section 4 presents and evaluates experimental results. Finally, we summarize our research and conclude the paper in Section 5.

\section{Generalized Discrete Radon Transform}

The Generalized Multi Directional Radon transform (GMDRT), as detailed in [13], is defined to detect from the initial image geometric objects by precisely locating its positions, its parameters and its rotation angles. The GMDRT method consists of projecting the image over parameterized curves $\phi$ that are rotated according to an angle $\theta$ and translated according to both the horizontal and the vertical axis as it is shown in figure1:
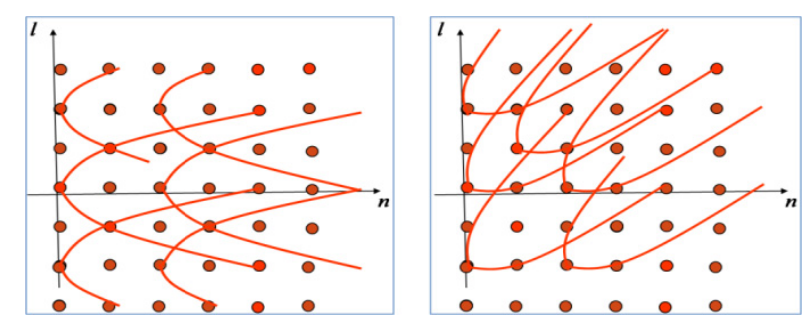

Fig. 1. (a): the parabolic curves $\phi(l)=l^{2}$ follow the horizontal direction (b):the rotated parabolic curves follow the $\pi / 4$ direction .

The GMDRT is an algebraic exactly invertible method inspired from [2] based on the multiplication of a selection matrix $R_{m, \theta}$ and an image column $I(n)$. The transform matrix $R_{m, \theta}$ is defined to select from a column $I(n)$, pixels belonging to a $\theta$ rotated curve. Let the following expression be the GMDRT formalism:

$$
y_{\theta}(n)=\sum_{m=-M}^{m=M} R_{m, \theta} \times I(n+m)=\left(\begin{array}{l}
y_{\theta}(-L, n) \\
. \\
y_{\theta}(0, n) \\
. . \\
y_{\theta}(L, n)
\end{array}\right) \text { and } y_{\theta}=\left(\begin{array}{l}
y_{\theta}(0) \\
y_{\theta}(1) \\
. . \\
. . \\
y_{\theta}(N-1)
\end{array}\right)
$$

Where $I(n)$ is a vector presenting a fixed column $n$ of the two dimensional image $I(l, n)$ with $l,-L \leq l \leq L$ and $n, 0 \leq n \leq N-1$ : 


$$
I(n)=\left(\begin{array}{l}
I_{0}(n) \\
\ldots \\
I_{l}(n) \\
\ldots \\
I_{L-1}(n)
\end{array}\right) \text { and the intire image as } I=\left(\begin{array}{l}
I(0) \\
I(1) \\
. \\
\\
I(N-1)
\end{array}\right)
$$

One condition on $I(n)$ is that it must be periodic, with period $N$, such that:

$$
I(n)=I(n+N)
$$

$M$ represents the number of neighboring vectors (i.e., columns of the image) either side of the input vector $I(n)$ involved in calculating $y_{\theta}(n)$ where $N=2 M+1$.

$\theta, \theta \in\left[0,2 \pi\left[\right.\right.$ denotes the projection angle of GMDRT. $y_{\theta}(j, n)$ presents the sum of the pixels centered on the curve starting at the position $(j, n)$ and rotated according to the angle $\theta . R_{m, \theta}$ are $(2 L+1) \times(2 L+1)$ matrices whose non-zero entries select which elements of $I(n+m)$ contribute to the projection $y_{\theta}(n)$. Each row $j,-L \leq j \leq L$ in $R_{m, \theta}$ samples the pixels from $I(n+m)$ belonging to the curve starting at the position $(j, n)$ in the image $I$. The principle of the transform is illustrated in the figure 2.The transform matrix $R_{m, \theta}$ is constructed as follows:

$$
R_{m, \theta}(j, l)=\delta\left(m^{\prime}-\phi\left(l^{\prime}\right)\right)
$$

where

$$
\left(l^{\prime}, m^{\prime}\right)= \begin{cases}R t_{\theta}^{-1}\left(<l-j>_{-(2 L+1)}, m\right) & \text { if } L<l-j \leq 2 L \\ R t_{\theta}^{-1}\left(<l-j>_{(2 L+1)}, m\right) & \text { if }-2 L \leq l-j<-L \\ R t_{\theta}^{-1}(l-j, m), & \text { if }-L \leq l-j \leq L\end{cases}
$$

Where $l,-L \leq l \leq L$ and $j,-L \leq j \leq L . \delta(\mu)$ is the kronecker delta function and $\langle\mu>\mathrm{x}$ denotes $\mu(\bmod \mathrm{x})$ since $l-j$ is restricted to be included in the $[-L, L]$ interval to avoid to exceed the image borders. $j$ presents the vertical translation step of the curve $\phi$ along y-axis. $R t_{\theta}^{-1}$ means the inverse plane rotation according to a certain angle $\theta$.More details about the GMDRT method is provided in [13] and it is to note that for $\mathrm{j}=0$ and for $\theta=0$, the GMDRT formalism is equivalent to the formalism proposed by Beylkin [2] where the curves are uniquely projected according to the horizontal direction and is not vertically translated. Figure 2 presents $R_{0,0}$ and $R_{l, 0}$ selecting pixels belonging to a parabolic curves from respectively the columns $I(0)$ and $I(1)$ and starting both at columns 0 . 

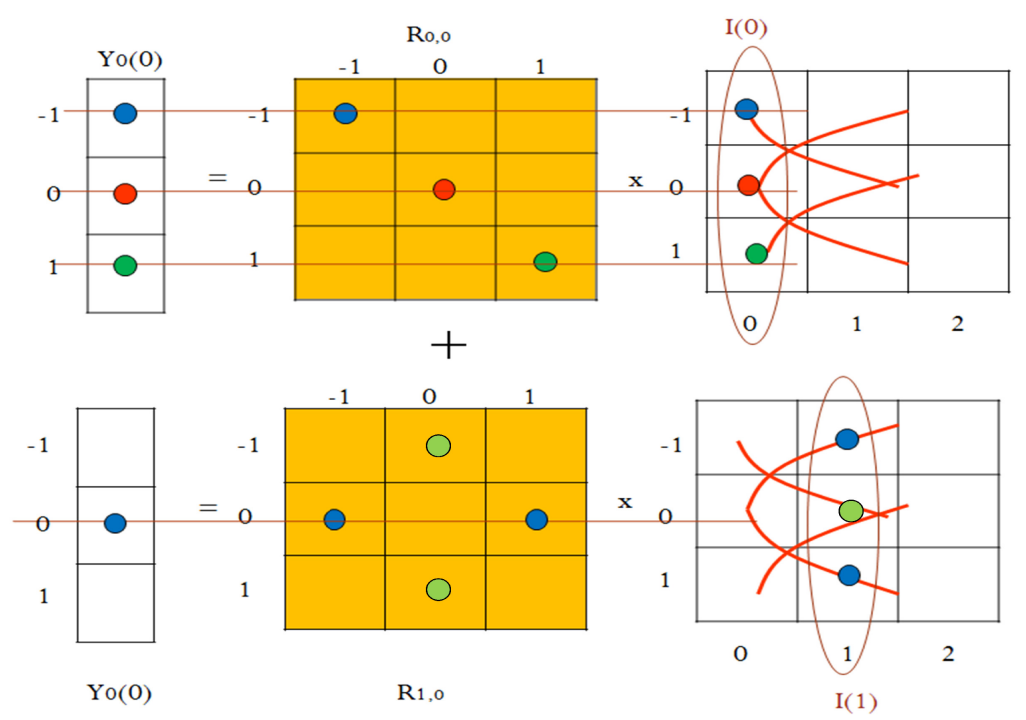

Fig. 2. A projection of horizontal parabolic curves $\phi(l)=l^{2}$ translated vertically, $\theta=0, M=1, L=1, N=3, n=0$.

The GMDRT then can be seen as a mapping between the image space and the Radon space. The coordinates of a point in the latter space correspond to the coordinates of an object oriented according to an angle $\theta$ in the image space. The intensity at that point corresponds to the amount of evidence for that object. The following figure represents the peaks resulting from the GMDRT on a simple image where rectangles are distributed and rotated in various directions. The peaks in the Radon space correspond to the coordinates of the upper left corner of the rectangle.

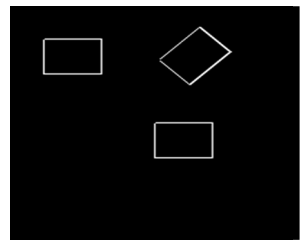

(a)

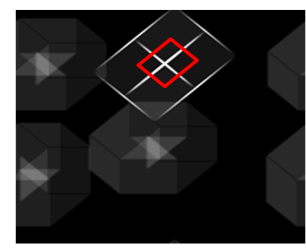

(b)

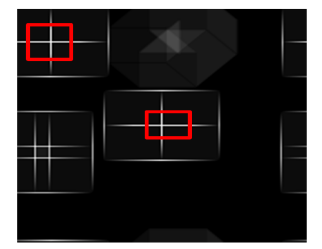

(c)

Fig. 3. (a): initial image, (b): The peak in red square resulting from projecting the initial image by $\pi / 4$-rotated rectangles. (c): The peaks projecting (a) with horizontal rectangles.

\section{Buildings Extraction}

The method developed in this study was applied to extract buildings from a Quickbird image of Strasbourg city with a spatial resolution 0.6 metre/pixel. The initial image is pretreated to remove noise. Then, we have extracted from the image edges by 
means of the Perwitt operator. After that, we have applied on the edge image the GMDRT approach and in final, we have extracted the local maximas to detect the peaks.

\subsection{Pretreatment Phase}

The initial image is treated by the multi-scale mathematical morphological filter. This filter [10] operates with four structure elements of various scales and was used to preserve building boundaries while removing noise such as thinner lines and spots. In addition, This operation decreases the spectral variability of the building regions.

\subsection{Extracting Contours}

We detect the edges of the original image by means of the Perwitt operator [14] which finds edges using the Perwitt approximation to the derivative. It returns edges at points where the gradient of the image is the maximum.

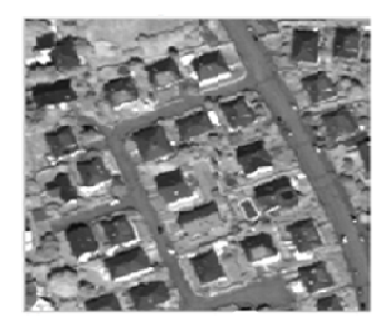

(a)

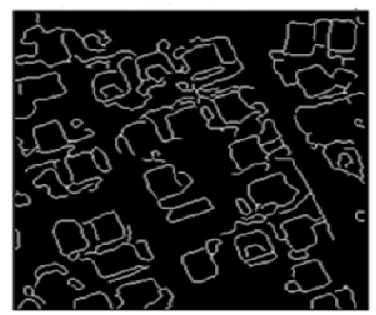

(b)

Fig. 4. (a) the initial image. (b) the contours image.

\subsection{Applying GMDRT}

We have applied iteratively the GMDRT transform on the contour image by projecting it with rectangular objects. For that, we vary at each iteration, the parameters i.e. the length $l$ and the width $w$ of the projected rectangles and its orientation angle $\theta$. Thus, the result of a GMDRT transform is the Radon images $y_{\theta, l, w}$. Taking in account the image resolution and the general information about buildings, we have varied $l$ and $w$ in the [19-32] interval and $\theta$ in $\left[0^{\circ}, 180^{\circ}\right.$ [ applied with $5^{\circ}$ step.

\subsection{Local Maximas}

$y_{\theta, l, w}$ is a two dimensional Radon space where each pixel denotes the projection result of a rectangle having as parameters $l, w$ and oriented according to $\theta$ angle. Therefore, a peak in the Radon image testifies of the coordinates and parameters of a building in that position. To detect the peaks in the Radon images, we have used a 
local maximas filter. In our tests, the size of the local maxima filter was fixed to be $21 \times 21$ taking in consideration the minimum distance between buildings. After selecting the local maximas in all $y_{\theta, l, w}$ images, we check the peaks coordinates. If more than one peak are at the same position or at a very approximate positions, we select the peak with the highest value.

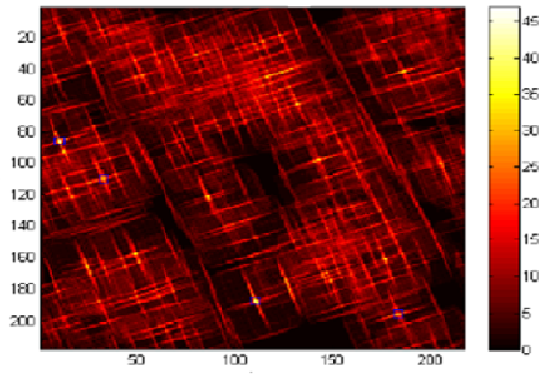

(a)

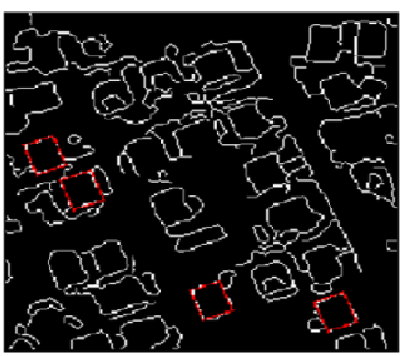

(b)

Fig. 5. A GMDRT projection via rectangles sized of $23 \times 20$ and rotated according to $20^{\circ}$ angle. (a)some of peaks in $y_{\pi / 4,23,20 .}$. b): The related rectangles in the contour image.

\section{$4 \quad$ Experiments}

To evaluate the efficiency of the proposed approach in the buildings extraction, we used a metric defined in [12]:

For a quantitative assessment:

$$
B E R=\frac{B C E}{T B}
$$

Where BER is the Building Extraction Rate, BCE is the Correctly Extracted Buildings number and TB is the total buildings number existing in the area of test.

And for a qualitative metric, we use these formalisms:

$$
\begin{gathered}
\text { exactness }=\frac{B C E}{B C E+F A} \\
\text { quality }=\frac{B C E}{B C E+B P E+B N E+F A}
\end{gathered}
$$

Where BPE is the number of Partially Extracted Buildings, BNE is the number of Not Extracted Buildings and FA is the False Alarms denoting the wrongly identified buildings. We applied our approach on five test images. We present here some of our results(Figure6 and Figure7).The table1 presents the measures of GMDRT on each of the five images. We have compared our results with those of three other approaches. The first was developed using the $R-\theta$ signature [15]which presents a new shape descriptor based on Classical Radon transform, the second " $D C B$ ”, extracts buildings with reference to geodatabase and prior knowledge[12] and the last method " $D R V$ " which detects buildings with the help of photometric, geometric and morphological informations[11]. The choice of these methods was guided by the similarity between their images characteristics and ours. 

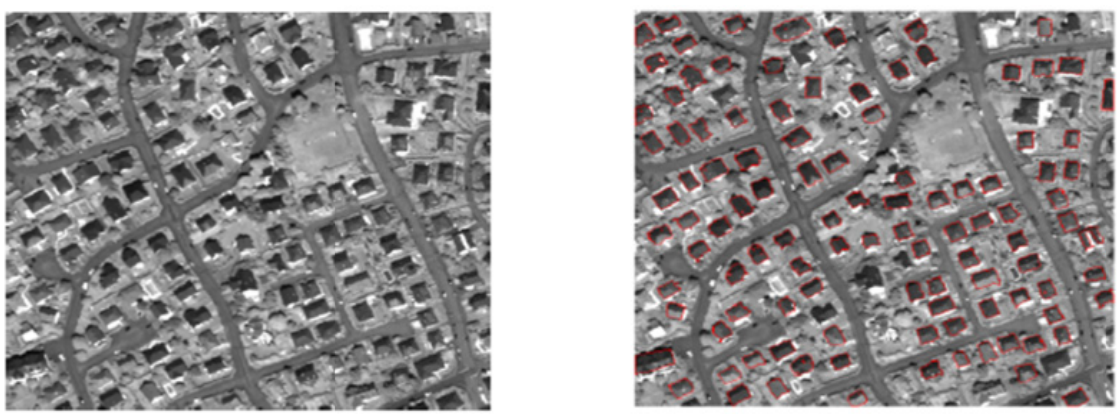

Fig. 6. On the left: initial image I1.on the right: the extracted buildings in red contours
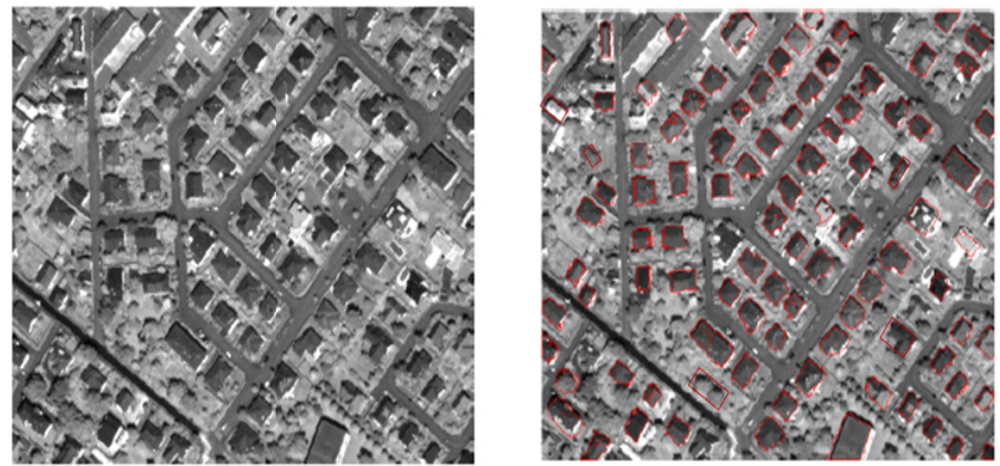

Fig. 7. On the left: initial image I2. on the right: the extracted buildings.

Table 1. (a) The measures of the GMDRT results. (b) The results rates of DCB,DRV, $R-\theta$ Signature and GMDRT methods.

\begin{tabular}{|l|c|l|l|l|l|l|}
\cline { 2 - 7 } \multicolumn{1}{c|}{} & II & I2 & I3 & I4 & I5 & Total \\
\hline BCE & 100 & 83 & 65 & 43 & I84 & 475 \\
\hline BPE & 2 & 4 & 0 & 0 & 2 & 8 \\
\hline BNE & 8 & 5 & 13 & 3 & 17 & 46 \\
\hline FA & 4 & 3 & 3 & 0 & 5 & 15 \\
\hline TB & 110 & 92 & 78 & 46 & 203 & 529 \\
\hline
\end{tabular}

(a)

\begin{tabular}{|c|l|l|l|}
\hline methods & BER(\%) & Exactness & Quality \\
\hline DCB & 94 & 0.903 & 0.855 \\
\hline DRV & 91 & 0.940 & 0.824 \\
\hline $\begin{array}{c}\text { R- } \theta \text { sig- } \\
\text { nature }\end{array}$ & 83 & 0.926 & 0.808 \\
\hline GMDRT & 89 & 0.969 & 0.873 \\
\hline
\end{tabular}

(b)

We show that the Buildings extraction rate of our method is less than the other DCB and DRV approaches but this result can be improved if the quality of the contour image is perfectly performed since the GMDRT process is closely related to the extraction contours results. In another hand, our method seems to be more perfect regarded to the quality and the exactness rates. In fact, the GMDRT method tends to limit the number of the buildings partially extracted and the false alarms. 


\section{Conclusion}

This paper describes the GMDRT approach and its application in building extraction. The results showed that our approach has good performance in detecting rectangular building shapes in the image but the user interfere to setting the dimensions interval of rectangles is a problem itself. In the future work we will try to make the Rectangular GMDRT fully automatic method in the way that the sizes of the rectangles in the image will be extracted with the help of the GMDRT itself.

\section{References}

1. Tofts, P.: The RadonTransform: Theory and implementation. Ph.D.Thesis (1996)

2. Beylkin, G.: Discrete Radon transform. IEEE Transactions of Acoustics, Speech and Signal Processing 35, 162-172 (1987)

3. Magli, E., Olmo, G., LoPresti, L.: Pattern recognition by means of the Radon transform and the continuous wavelet transform. Signal Processing 73 (1999)

4. Milanfar, P.: A model of the effect of image motion in the Radon transform domain. IEEE Transactions on Image Processing 8, 1276-1281 (1999)

5. Shepp, L.A., Krustal, J.B.: Computerized tomography: The new medical X-ray technology. Am. Math. Monthly 85, 420-439 (1978)

6. Courmontagne, P.: An improvement of ship wake detection based on the radon transform. Signal Processing 85 (2005)

7. Krishnaveni, M., Kumar Thakur, S., Subashini, P.: An optimal method for wake detection in SAR images using Radon transformation combined with wavelet filters. International Journal of Computer Science and Information Security 6, 066-069 (2009)

8. Zhang, Q., Couloigner, I.: Accurate Centerline Detection and Line Width Estimation of Thick Lines using the Radon Transform. IEEE Transactions On Image Processing 16, 310-316 (2007)

9. Wang, L., Hao, Y.: Radon Transform and Forstner Operator Applying in Buildings Contour Extraction. In: Sixth International Conference on FSKD, pp. 415-419 (2009)

10. Mukhopadhyay, S., Chanda, B.: An edge preserving noise smoothing technique using multi-scale morphology. Signal Processing 82, 527-544 (2002)

11. Lhomme, S., He, D.C., Weber, C., Morin, D.: A new approach to building identification from very-high-spatial-resolution images. International Journal of Remote Sensing 30, 1341-1354 (2009)

12. Bouziani, M., Goita, K., He, D.-C.: Automatic change detection of buildings in urban environment from very high spatial resolution images using existing geodatabase and prior knowledge. ISPRS J. of Photogrammetry and Remote Sensing 65, 143-153 (2010)

13. Elouedi, I., Fournier, R., Nait-Ali, A., Hammouda, A.: Generalized Multi Directional Discrete Radon Transform. Signal Processing (paper in revision)

14. Zhang, W., Bergholm, F.: Multi-scale blur estimation and edge type classification for scene analysis. International Journal of Computer Vision 24, 219-250 (1997)

15. Hamouda, A., Rojbani, H., Elouedi, I.: A new shape descriptor based on the Radon transform: the $\mathrm{R} \theta$-signature. Accepted paper in International Conference on Signal, Image Processing and Applications, ICSIA (2011) 